\title{
X-ray structural analysis of crystalline materials at the XSA/Belok beamline at synchrotron radiation source of the Kurchatov institite
}

\author{
Lazarenko V.A. ${ }^{1}$, Zubavichus Y.V. ${ }^{2}$, Dorovatovsky P.V. ${ }^{1}$, Svetogorov R.D. ${ }^{1}$ \\ ${ }^{I}$ Naional Research Center "Kurchatov institute", Russia, Moscow, \\ ${ }^{2}$ Boreskov Institute of Catalysis SB RAS,
}

Vladimir.a.lazarenko@gmail.com

At the moment, single-crystal diffraction remains the most popular and widespread method for solving spatial structures of varying complexity for coordination chemistry and biology. Using a synchrotron radiation source for conducting this type of experiment allows one to achieve high resolution in the shortest time.

Despite the daily increase in demand for solving coordination chemistry problems and, accordingly, working with small molecules, the number of synchrotron beamlines for single crystal diffraction by small molecules is quite small, and protein crystallography stations are given priority.

The main ones are stations I19 on Diamond, 11.3.1 on ALS, BM01 on ESRF, XRD on Elettra, while the quantity of beamlines specialized for macromolecular objects are an order of magnitude larger. This is mainly due to the popularity of biological problems in the modern world, as well as the peculiarities of macromolecule crystals, because of which data collection at a laboratory source becomes almost impossible.

To make it easier for Russian users and provide an additional opportunity for foreign users to access this type of synchrotron beamline and to quickly collect high-resolution diffraction data from a wide range of samples, one of the installations of the Kurchatov synchrotron radiation source was optimized for working with crystalline samples in mass flow measurements, which allowed it to become a device that has no analogues in Russia for conducting this type of experiment [1]. Subsequently, to increase the quality of the data obtained, the diffractometer from the Belok station was transferred to the XSA beamline (Fig. 1). In addition, due to an increase in the intensity of the photon beam and the quality of the data collected, the number of experiments on the study of protein crystals has increased several times.

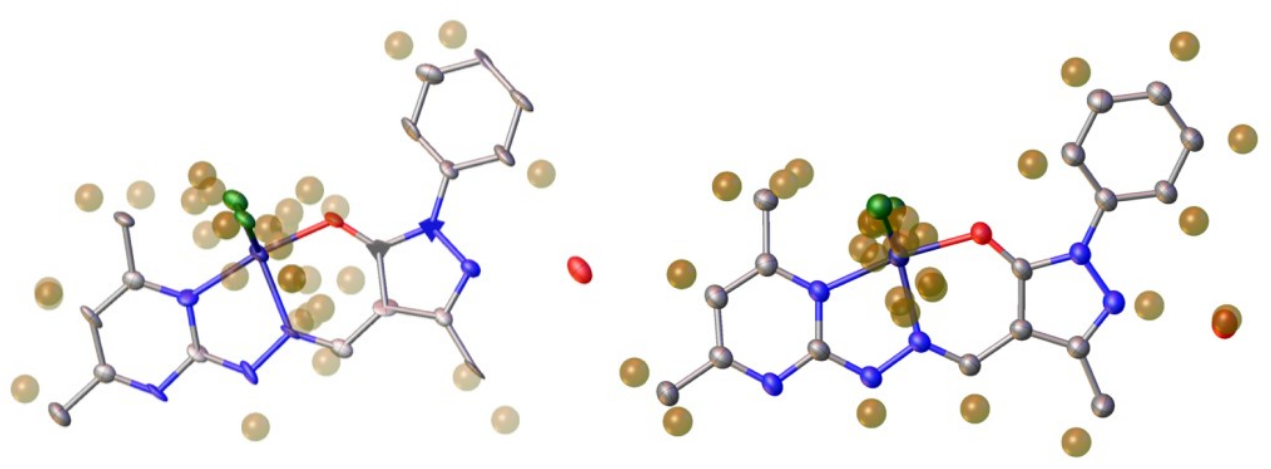

Figure 1. Image of structural models of a complex of hetarylhydrazone with copper chloride obtained from the results of the experiment at Belok beamline (left) and XSA/Belok beamline (right), as well as images of residual electron density peaks

Raytracing of the XSA station was carried out and a noticeable increase in the photon flux on the sample was shown in comparison with the Belok station, where the hight-throutput single-crystal small-molecular crystallography experiments had previously been started [2]. The optimization of all stages of the structure solution and the demonstration of the quality of the data obtained were carried out using various classes of compounds as an example.

[1] Lazarenko V.A., Dorovatovskii P.V., Zubavichus Y.V., Burlov A.S., Koshchienko Y.V.,Vlasenko V.G., Khrustalev V.N., Crystals. 2017. V. 7. P 325-1-19.

[2] Roman D. Svetogorov, Pavel V. Dorovatovskii, Vladimir A. Lazarenko, Crystal Research \& Technology, 2020, in press

Keywords: X-ray single crystal diffraction, small molecules, diffraction beamline, high-throughput crystallography 